\title{
A gas trapping method for high-throughput metabolic experiments
}

\author{
James R. Krycer ${ }^{1}$, Ciana Diskinn', Marin E. Nelson ${ }^{1}$, Xiao-Yi \\ Zeng $^{1}$, Daniel J. Fazakerley ${ }^{1}$, and David E. James ${ }^{1,2}$ \\ ${ }^{1}$ School of Life and Environmental Sciences, Charles Perkins \\ Centre, The University of Sydney and 'Sydney Medical School, \\ The University of Sydney
}

BioTechniques 64:27-29 (January 2018) doi 10.2144/000114629

Keywords: gas trap; adipocyte; cell metabolism; carbon dioxide; hydrogen sulfide

Supplementary material for this article is available at www.BioTechniques.com/article/114629.

Research into cellular metabolism has become more high-throughput, with typical cell-culture experiments being performed in multiwell plates (microplates). This format presents a challenge when trying to collect gaseous products, such as carbon dioxide $\left(\mathrm{CO}_{2}\right)$, which requires a sealed environment and a vessel separate from the biological sample. To address this limitation, we developed a gas trapping protocol using perforated plastic lids in sealed cell-culture multiwell plates. We used this trap design to measure $\mathrm{CO}_{2}$ production from glucose and fatty acid metabolism, as well as hydrogen sulfide production from cysteine-treated cells. Our data clearly show that this gas trap can be applied to liquid and solid gas-collection media and can be used to study gaseous product generation by both adherent cells and cells in suspension. Since our gas traps can be adapted to multiwell plates of various sizes, they present a convenient, cost-effective solution that can accommodate the trend toward high-throughput measurements in metabolic research.

Over the past century, research into cellular metabolism has involved measuring how cells generate metabolites of interest in response to specific perturbations. This approach has become more high-throughput with advances in tissue-culture techniques, such that experiments are now typically performed in multiwell plates (microplates). In this format, it is easy to collect aqueous metabolites, both from the cells and the media. However, these plates are designed to facilitate gaseous exchange within a tissue-culture incubator, which presents a challenge when attempting to collect and assay gaseous products. Here, we present a convenient, cost-effective solution that can be applied to multiwell plates of various sizes.

Initially, we established this gas-trapping technique to measure the oxidation of radiolabelled glucose. Following treatment, the biological sample (media containing cells or tissue slices) is quenched by a strong acid, and the oxidation product $\left(\mathrm{CO}_{2}\right)$ is collected in an alkali solution ( $\mathrm{NaOH})$. To minimize contamination, the alkali solution is separated from the biological sample by either a hanging well suspended above the biological sample $(1,2)$ or in a center well (3). This was classically performed with biological samples in separate vials or flasks. We found that a simple solution for adapting this strategy to a multiwell plate format involved drilling a hole into the lids of $15-\mathrm{mL}$ centrifuge tubes (protocol included as Supplementary Material). When positioned upside-down, these perforated lids fit well into the top of each well in a 12-well plate, hold an adequate quantity of alkali solution, and minimize liquid handling (Figure 1, A and B). Likewise, 6-mL scintillation vial lids can be modified similarly for a 24-well plate format (Supplementary Material). As a proofof-principle, we treated 3T3-L1 adipocytes with insulin in the presence of a $\left[\mathrm{U}-{ }^{14} \mathrm{C}\right]$-glucose tracer. The wells were sealed with Parafilm after setting up the gas-trap and again following acidification of the well contents (cells and media) with perchloric acid (Supplementary Material). After $1 \mathrm{~h}$, the alkali solution was assayed for ${ }^{14} \mathrm{C}$ content. Using this protocol, we detected insulinresponsive glucose oxidation (Figure 1C). Thus, a perforated plastic lid can serve as a suitable gas trap.

Next, we investigated the sealing method for this multiwell plate format. Each well needs to be adequately sealed to ensure the $\mathrm{CO}_{2}$ produced does not escape collection. A previous study sealed each individual well with rubber hosing (4); however, this impairs visibility when injecting acid into the well, so we used Parafilm as a substitute seal. A potential caveat with our approach is that the seal is broken during acid quenching, which may result in significant $\mathrm{CO}_{2}$ loss. To test for this potential loss, we measured ${ }^{14} \mathrm{C}-\mathrm{CO}_{2}$ production from insulin-stimulated 3T3-L1 adipo-

\section{METHOD SUMMARY}

We developed a gas trapping protocol for cell metabolism experiments using perforated plastic lids in sealed tissue-culture multiwell plates. These traps can hold either liquid or solid collection media and are adaptable to multiple cell types. 
A

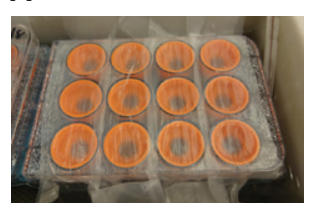

B

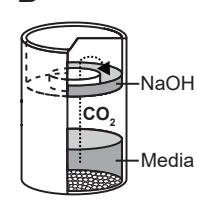

D
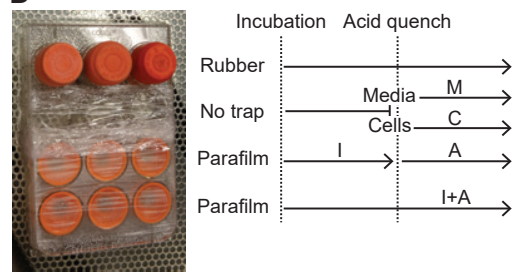

$\mathrm{E}$

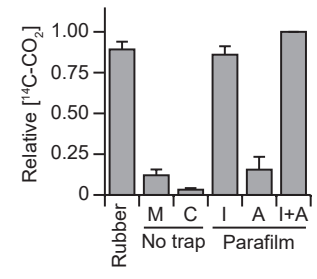

Figure 1. A high-throughput gas trap system constructed using perforated plastic lids in a sealed multiwell plate. (A) Photograph of the gas trap system. (B) Schematic of the gas trap system, adapted for collecting $\mathrm{CO}_{2}$ using an alkali solution $(2 \mathrm{M} \mathrm{NaOH})$ dispensed into the trap above the cells. (C) 3T3-L1 adipocytes were grown and differentiated as previously described (9). Cells were starved at $37^{\circ} \mathrm{C}$ in DMEM supplemented with $0.2 \%$ (w/v) BSA, $30 \mathrm{mM}$ HEPES ( $\mathrm{pH}$ 7.4), $25 \mathrm{mM}$ glucose, $1 \mathrm{mM}$ glutamine, and $1 \mathrm{mM}$ GlutaMax (Life Technologies) for $2 \mathrm{~h}$. Cells were then incubated at $37^{\circ} \mathrm{C}$ for $1 \mathrm{~h}$ with or without $100 \mathrm{nM}$ insulin in the same media, except supplemented with $10 \mathrm{mM}$ glucose and $1 \mu \mathrm{Ci} / \mathrm{mL}\left[\mathrm{U}-{ }^{14} \mathrm{C}\right]$-glucose (Perkin Elmer), in the presence of gas traps containing $\mathrm{NaOH}$ solution. Cells were quenched with 0.2 vol of $1 \mathrm{M}$ perchloric acid (Supplementary Material), and the plate was resealed. After $1 \mathrm{~h}$ incubation at room temperature, the ${ }^{14} \mathrm{C}$ content in the $\mathrm{NaOH}$ solution was measured by liquid scintillation counting. A separate batch of cells (treated identically but without acid quenching) was lysed in PBS with $1 \%$ Triton X-100, and cellular protein was quantified using the BCA assay (Pierce). Glucose oxidation $\left({ }^{14} \mathrm{C}-\mathrm{CO}_{2}\right)$ was normalized to protein content. Data are presented as mean $\pm \mathrm{SEM}$ from three separate experiments. (D) Photograph and schematic for the experiment performed in (E). (E) 3T3-L1 adipocytes were treated with insulin as described in $(C)$ with: no modifications (Parafilm, $\mathrm{I}+\mathrm{A})$; collection of $\mathrm{NaOH}$ solution after the treatment incubation (Parafilm, I), and replacement with fresh $\mathrm{NaOH}$ solution, which was collected after acid quenching (Parafilm, A); a rubber seal instead of a Parafilm seal (Rubber); no gas trap during the treatment incubation, with the media (No trap, M) and cells (No trap, C) separately quenched with acid and subsequently incubated with vial-based (10) and Parafilm-sealed gas traps (similar to Parafilm wells) respectively. To ensure a fair comparison between conditions, the times for treatment and post-quench incubations were the same across all conditions ( $1 \mathrm{~h}$ each), and manipulations prior to each of these incubations took less than $5 \mathrm{~min}$ in total to perform. ${ }^{14} \mathrm{C}$ content (DPM) was blank-corrected to naïve $\mathrm{NaOH}$ solution and measurements were reported relative to the Parafilm I+A condition. Data are presented as mean $\pm S D$ from at least two separate experiments per condition.

cytes where the gas trap was sealed with either Parafilm or a rubber cap (Figure 1D). To explore the importance of the seal, we also included wells without a trap during the treatment incubation, after which the cells and media were separately acidified for $\mathrm{CO}_{2}$ collection. Furthermore, to determine the importance of the acidification step, we collected the alkali solution at the end of the treatment incubation and replenished the gas trap with fresh alkali solution before acidification. We found that Parafilm performed similarly to the rubber seal (Figure 1E; Rubber versus Parafilm I+A), such that the majority of $\mathrm{CO}_{2}$ was trapped during the treatment incubation period (Figure 1E; Parafilm I versus Parafilm I+A). The acidification step provided a slight improvement in signal (Figure 1E; Parafilm A). Accordingly, we found that most of the signal was lost if the trap was absent during the incubation
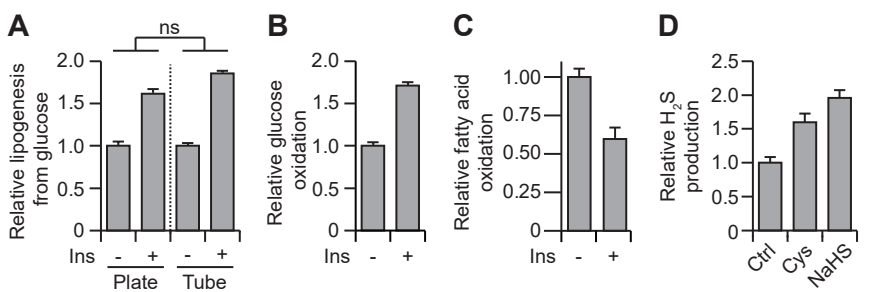

Figure 2. The gas trap system can be applied to cells in suspension and to solid collection media. (A) Primary adipocytes were isolated from epidydimal fat pads of C57BL6/J mice as previously described (5). Primary adipocytes were incubated for $1 \mathrm{~h}$ with or without $20 \mathrm{nM}$ insulin (Ins) in Krebs-Ringer bicarbonate/HEPES buffer (5) supplemented with $10 \mathrm{mM}$ glucose and $2 \mu \mathrm{Ci} / \mathrm{mL}\left[\mathrm{U}-{ }^{14} \mathrm{C}\right]$-glucose. Incubations were performed either in Parafilm-sealed polystyrene multiwell plates (Plate) or polypropylene tubes (Tube). Following incubation, lipids were isolated by methanol/chloroform extraction (11) and saponified (12). Esterified lipids were isolated by petroleum ether extraction and quantified by liquid scintillation counting (12). ${ }^{14} \mathrm{C}$ content was reported relative to that of the cells without insulin within each condition. Data are presented as mean \pm SEM from at least three separate experiments per condition. A two-way ANOVA showed the effect of insulin treatment to be significant $(P<0.05)$, but neither the effect of vessel type (Plate, Tube) nor the interaction between insulin treatment and vessel type was significant $(P>0.05$, labeled "ns"). (B) Primary adipocytes were isolated and treated as in (A), except in sealed multiwell plates containing the $\mathrm{CO}_{2}$ gas trap system described in Figure $1, \mathrm{~A}$ and $\mathrm{B}$. Glucose oxidation $\left({ }^{14} \mathrm{C}-\mathrm{CO}_{2}\right)$ was measured after acid-quenching by liquid scintillation counting of the alkali solution as in (B) and reported relative to the cells without insulin. Data are presented as mean \pm SEM from four separate experiments. (C) Primary adipocytes were treated and assayed as in (B), except the buffer was supplemented with $10 \mathrm{mM}$ glucose and $125 \mu \mathrm{M}$ palmitate with trace amounts of $\left[1-{ }^{14} \mathrm{C}\right]$-palmitate (Perkin Elmer) conjugated to BSA. The final concentration of palmitate tracer in the assay buffer was $10 \mu \mathrm{Ci} /$ $\mathrm{mL}$, and albumin was $0.25 \%(\mathrm{w} / \mathrm{v})$. Fatty acid oxidation $\left({ }^{14} \mathrm{C}-\mathrm{CO}_{2}\right)$ was measured as described in Figure 1B and reported relative to the cells without insulin. Data are presented as mean \pm SEM from four separate experiments. (D) HEK-293E cells were cultured as described previously (13). Cells were then treated without (control, Ctrl), with $50 \mathrm{mM}$ cysteine (Cys), or with $0.5 \mathrm{mM} \mathrm{NaHS}$ for $24 \mathrm{~h}$ in DMEM supplemented with 10\% (v/v) FBS (Life Technologies), $25 \mathrm{mM}$ glucose, $2 \mathrm{mM}$ glutamine, $30 \mathrm{mM}$ HEPES ( $\mathrm{pH}$ 7.4), and $1 \mathrm{mM}$ bicarbonate. During this treatment period, cells were incubated at $37^{\circ} \mathrm{C}$ in sealed multiwell plates with gas traps containing sterile agar containing alkaline zinc acetate (Supplementary Material). Following incubation, the agar was assayed for $\mathrm{H}_{2} \mathrm{~S}$ content by spectrometry, as described previously (7). $\mathrm{H}_{2} \mathrm{~S}$ content was reported relative to the untreated cells. Data are presented as mean \pm SEM, from four separate experiments.

period (Figure 1E; No trap versus Parafilm I+A). Taken together, these results justified the use of a Parafilm seal for the gas trap and demonstrated the importance of collecting $\mathrm{CO}_{2}$ during the treatment incubation in addition to after the acidquenching step. Although acid treatment only provided a slight improvement in gas collection, the investigation of the acid-soluble metabolites could be an important justification for the acidification step in the design of metabolic experiments.

Following optimization in 3T3-L1 adipocytes, an adherent cell-line, we next applied this experimental setup to primary mouse adipocytes, which were maintained in suspension. For this application, primary adipocytes were incubated in sealed polystyrene multiwell plates on a rocking platform in a $37^{\circ} \mathrm{C}$ incubator. To our knowledge, primary adipocytes have not been previously incubated in this format; therefore, we compared their insulin sensitivity to cells incubated in polypropylene tubes in a shaking water bath (5). Using lipogenesis from ${ }^{14} \mathrm{C}$-glucose as a readout for insulin sensitivity, we found 
that primary adipocytes were equally responsive to insulin in either format (Figure 2A). Using our gas trap, we detected insulin-stimulated glucose oxidation in primary adipocytes (Figure 2B) with effect sizes similar to those previously reported for cells grown in individual flasks (1). Furthermore, we observed that insulin suppressed oxidation of ${ }^{14} \mathrm{C}$-labeled palmitate (Figure 2C), with a carnitine-palmitoyltransferase-1 inhibitor (etomoxir, $20 \mu \mathrm{M}$ ) reducing palmitate oxidation by $77 \%$ (data not shown). These results demonstrate that our gas trap can be used to measure the oxidation of different substrates in suspended cells in a high-throughput fashion.

Finally, we tested the application of solid gas-collection media. As proof of principle, we chose to measure cellular $\mathrm{H}_{2} \mathrm{~S}$ production using solid agar $(6,7)$. Agar containing alkaline zinc acetate was applied to the gas trap. This was used to measure $\mathrm{H}_{2} \mathrm{~S}$ production from HEK-293E cells treated with or without cysteine, which generates $\mathrm{H}_{2} \mathrm{~S}$ as part of its catabolism. Following treatment, the agar was assayed for $\mathrm{H}_{2} \mathrm{~S}$ content by spectrometry (7). As a positive control, cells were treated with NaHS. We found that these gas traps could detect $\mathrm{H}_{2} \mathrm{~S}$, and as expected, cysteine treatment increased $\mathrm{H}_{2} \mathrm{~S}$ production (Figure 2D). Although unconventional, an agar-based method (8) could also be used for trapping $\mathrm{CO}_{2}$. Overall, these gas traps can be used with solid media to collect gaseous products.

Here, we present a convenient and cost-effective method for gas collection via liquid or solid media. Our approach is compatible with adherent and suspension cell cultures, and it can be adapted to either 12- or 24-well multiwell plate formats, thus enabling metabolic experiments to be performed in a high-throughput manner.

\section{Author contributions}

J.R.K., D.J.F., and D.E.J. conceived the study. J.R.K. and C.D. designed, performed, and analyzed data for cell-culture experiments. J.R.K., M.E.N., and X.Y.Z. designed, performed, and analyzed data for primary adipocyte experiments. J.R.K. and D.E.J. wrote the manuscript. All authors edited the manuscript. D.J.F. and D.E.J. supervised the study.

\section{Acknowledgments}

This work is supported by National Health and Medical Research Council (NHMRC) program grants (GNT1061122 and GNT1086850 for D.E.J.). J.R.K. is an NHMRC Early Career Fellow (APP1072440). D.E.J. is an NHMRC Senior Principal Research Fellow (APP1019680). The contents of the published material are solely the responsibility of the authors and do not reflect the views of the NHMRC.

\section{Competing interests}

The authors declare no competing interests.

\section{References}

1. Rodbell, M. 1964. Metabolism of isolated fat cells. I. Effects of hormones on glucose metabolism and lipolysis. J. Biol. Chem. 239:375-380.
2. MacDonnell, P. and O. Greengard. 1975. The distribution of glutamate decarboxylase in rat tissues; isotopic vs fluorimetric assays. J. Neurochem. 24:615-618.

3. Liu, L., S. Shah, J. Fan, J.O. Park, K.E. Wellen, and J.D. Rabinowitz. 2016. Malic enzyme tracers reveal hypoxia-induced switch in adipocyte NADPH pathway usage. Nat. Chem. Biol. 12:345-352.

4. Bauer, D.E., J.G. Jackson, E.N. Genda, M.M. Montoya, M. Yudkoff, and M.B. Robinson. 2012. The glutamate transporter, GLAST, participates in a macromolecular complex that supports glutamate metabolism. Neurochem. Int. 61:566-574.

5. Simpson, I.A., D.R. Yver, P.J. Hissin, L.J. Wardzala, E. Karnieli, L.B. Salans, and S.W. Cushman. 1983. Insulin-stimulated translocation of glucose transporters in the isolated rat adipose cells: characterization of subcellular fractions. Biochim. Biophys. Acta 763:393-407.

6. Lopez del Castillo Lozano, M., R. Tache, P. Bonnarme, and S. Landaud. 2007. Evaluation of a quantitative screening method for hydrogen sulfide production by cheese-ripening microorganisms: the first step towards I-cysteine catabolism. J. Microbiol. Methods 69:70-77.

7. Kartha, R.V., J. Zhou, L.B. Hovde, B.W. Cheung, and H. Schroder. 2012. Enhanced detection of hydrogen sulfide generated in cell culture using an agar trap method. Anal. Biochem. 423:102-108.

8. Wang, H.J., Y.H. Jo, D.S. An, J.W. Rhim, and D.S. Lee. 2015. Properties of agar-based $\mathrm{CO}_{2}$ absorption film containing $\mathrm{Na}_{2} \mathrm{CO}_{3}$ as active compound. Food Packaging and Shelf Life 4:36-42.

9. Fazakerley, D.J., S. Naghiloo, R. Chaudhuri, F. Koumanov, J.G. Burchfield, K.C. Thomas, J.R. Krycer, M.J. Prior, et al. 2015. Proteomic Analysis of GLUT4 Storage Vesicles Reveals Tumor Suppressor Candidate 5 (TUSC5) as a Novel Regulator of Insulin Action in Adipocytes. J. Biol. Chem. 290:23528-23542.

10. Hoy, A.J., C.R. Bruce, A. Cederberg, N. Turner, D.E. James, G.J. Cooney, and E.W. Kraegen. 2007. Glucose infusion causes insulin resistance in skeletal muscle of rats without changes in Akt and AS160 phosphorylation. Am. J. Physiol. Endocrinol. Metab. 293:E1358-E1364.

11. Folch, J., M. Lees, and G.H. Sloane Stanley. 1957. A simple method for the isolation and purification of total lipides from animal tissues. J. Biol. Chem. 226:497-509.

12. Stansbie, D., R.W. Brownsey, M. Crettaz, and R.M. Denton. 1976. Acute effects in vivo of anti-insulin serum on rates of fatty acid synthesis and activities of acetyl-coenzyme A carboxylase and pyruvate dehydrogenase in liver and epididymal adipose tissue of fed rats. Biochem. J. 160:413-416.

13. Yang, G., D.S. Murashige, S.J. Humphrey, and D.E. James. 2015. A Positive Feedback Loop between Akt and mTORC2 via SIN1 Phosphorylation. Cell Reports 12:937-943.

Received 10 October 2017; accepted 05 December 2017.

Address correspondence to David E. James, Charles Perkins Centre, The University of Sydney, Sydney, New South Wales 2006, Australia. E-mail: david.james@sydney.edu.au

To purchase reprints of this article, contact: biotechniques@fosterprinting.com 